\title{
A Fast Recognition Method for Pose and Illumination Variant Faces on Video Sequences
}

\author{
Mahesh Prasanna $\mathrm{K}^{1}$, Nagaratna Hegde ${ }^{2}$ \\ ${ }^{\prime}$ (ISE, Alva's Institute of Engineering \& Technology, India) \\ ${ }^{2}$ (CSE, Vasavi College of Engineering, India)
}

\begin{abstract}
Video face recognition is a widely used method in which security is essential that recognizes the human faces from subjected videos. Unlike traditional methods, recent recognition methods consider practical constraints such as pose and illumination variations on the facial images. Our previous work also considers such constraints in which face recognition was performed on videos that were highly subjected pose and illumination variations. The method asserted good performance however; it suffers due to high computational cost. This work overcomes such drawback by proposing a simple face recognition technique in which a cost efficient Active Appearance Model (AAM) and lazy classification are deployed. The deployed AAM avoids nonlinear programming, which is the cornerstone for increased computational cost. Experimental results prove that the proposed method is better than the conventional technique in terms of recognition measures and computational cost.
\end{abstract}

Keywords - Active Appearance Model (AAM), lazy classification, shape, model, appearance, recognition, computation.

\section{INTRODUCTION}

Face recognition can be termed as a technique of differentiating faces or confirming one or more individuals in a particular still or video image using a stored database [1] [2] [3]. It finds numerous applications in surveillance, human-computer interactions, authentication and security [4]. It is broadly classified into two types namely geometric feature-based and appearance-based [6]. The facial parts are considered as geometrical parameters and are utilized by geometric feature-based methods, for instance, elastic bunch graph matching [7] and active appearance model [8], while in appearance-based methods intensity or intensity-derived parameters are utilized [1] [17]. In a given video fragment, the process that is performed is associated with the image and is repeated for every frame. For this reason, we refer some face recognition approaches for a given image also when discussing about the recognition technique in video. The primary two stages of a face recognition technique is face detection and face identification [4]. Initially in the face detection stage, face images present in a given input image are located. Then to recognize the registered individuals of the system, faces located in the input image are then used by the face identification stage. This shows the sensible significance of having both face detection algorithms and face identification algorithms [13].

In face recognition, the foremost concerned characteristics are variations in illumination, pose, identity [5], facial expression, hair style, aging, make-up, scale etc,. The variation is challenging under severe illumination condition even for humans accurate recognition of faces because the same person appears extremely different [12]. As a solution, to overcome the problem and to manage pose variations in face recognition view-based method is principally used. In this method, the images are captured from diverse view angles to recognize the face images of the persons [15] [16]. Using the images of the same view an eigen space model is constructed for each view. By using the view-specific eigen model, a person in a different pose can be recognized effectively [14].

Only few years before the true video based face recognition methods that consistently use both spatial and temporal information began in which recognition of faces from video sequence still requires more recognition which is a direct extension of still-image-based recognition [9]. A typical video-based face recognition system determines the face regions automatically by extracting features from the video and distinguishing facial identities, which is often a difficult task [10]. In contrast to this, utilizing motion as a cue identification of the segmentation of a moving person is easier if a video sequence is available. Apart from this, a range of various factors like illumination, pose, identity, facial expression, hair style, aging, make-up, scale etc. have been focused in the previous research works. However, illumination and pose are the two major factors that influence face recognition.

The chief limitation of the pose-invariance recognition approach is the necessity to achieve and accumulate a large number of views for each face. This technique is unsuitable for conditions where only a small number of views of the face to be recognized are available. One of the most challenging problems is varying illumination and the most important drawback of illuminations is computational cost. To address and 
solve these problems, various algorithms have been developed for face recognition. A review on handful of research works has been presented and the problems are addressed in the following Section. The proposed technique to solve the addressed problem is detailed in Section 3. The results are discussed in Section 4 and Section 5 concludes the paper.

\section{RELATED WORKS}

S.Venkatesan and S.SrinivasaRaoMadane [18] have proposed a face recognition system. The system used Genetic and Ant colony Optimization algorithm to identify faces in images and video tracks faces, distinguish faces from galleries of well-known people. YileiXuet al. [19] have proposed a framework called analysis-by-synthesis framework. In the framework the face was recognized from video sequences under various facial pose and in lighting situation. Connolly, J.F et al. [20] proposed an adaptive classification system (ACS) for video-based face recognition. The system was a combination of fuzzy ARTMAP neural network classifier, dynamic particle swarm optimization (DPSO) algorithm, and a long term memory (LTM).

Unsang Park et al. [21] introduced the adaptive use of multiple face matchers to improve the performance of face recognition in video. In their method, active appearance model (AAM) and a Factorization based 3D face reconstruction technique were exploited to estimate the active information of facial poses in different frames. Huifuang $\mathrm{Ng}$ et al. [14] proposed an approach, which was not much sensitive to direction of views and required only one sample view per person. Xiujuan Chai, Shiguang Shan et al. [22] proposed a Locally Linear regression (LLR) method in which an essential frontal view is generated from a given non frontal face image. In order to accomplish this, they exploited linear mapping and the assessment of the linear mapping was formulated as a forecasting problem.

OgnjenArandjelovicet al. [23] presented a recognition method based on simple image processing filters to produce a single matching score between two different faces. They implicitly estimated the difference of the illumination conditions between query input and training dataset in the method. Sheikh et. al. [24] proposed a framework for human face retrieval in which face detection was performed using Viola and Jones frontal detector and the features were extracted using fast Haar transformation. Sheikh et. al. [25] converts the query image to a 3D- ellipsoid model based on the viola and jones frontal face detector. They used chamfer distance measure to performing recognition.

The review of works clearly interprets that the video faces are highly variable, deformable objects, and different appearances in frame depending on pose, lighting, expression, and the identity of the person. Besides that, the frame can have different backgrounds, differences in image resolution, contrast, brightness, sharpness, and color balance. This means that interpretation of such video face requires the ability to understand this variability in order to extract useful information and this extracted information must be of some manageable size. However, the recent related works have focused on such practical constraints such as pose and illumination variations of the facial images. In our previous work, we had developed a solution for considering both pose and illumination variation, however the methodology is highly complex despite it performed well.

In this work, we intend to propose a simple face recognition technique, which is simpler in terms of computational complexity, using Active Appearance Model (AAM). Active Appearance Model is well known for its differentiation and integration ability under different poses of subjected faces. Conventionally, the AAM is exploited along with a non-linear programming to extract features. The extracted features require a classifier; mostly an intelligent classifier is required for further recognition process. This again increases the computational complexity despite the features that distinguish pose variations. In order to avoid such complexities, we propose active appearance features without incorporation of nonlinear programming and a lazy classifier to training complexities.

\section{Proposed Face Recognition Technique}

The proposed technique is comprised of two stages, (i) Development of Feature Library and (ii) Recognition Stage. A high level block diagram of the proposed face recognition system is given in Figure 1. 


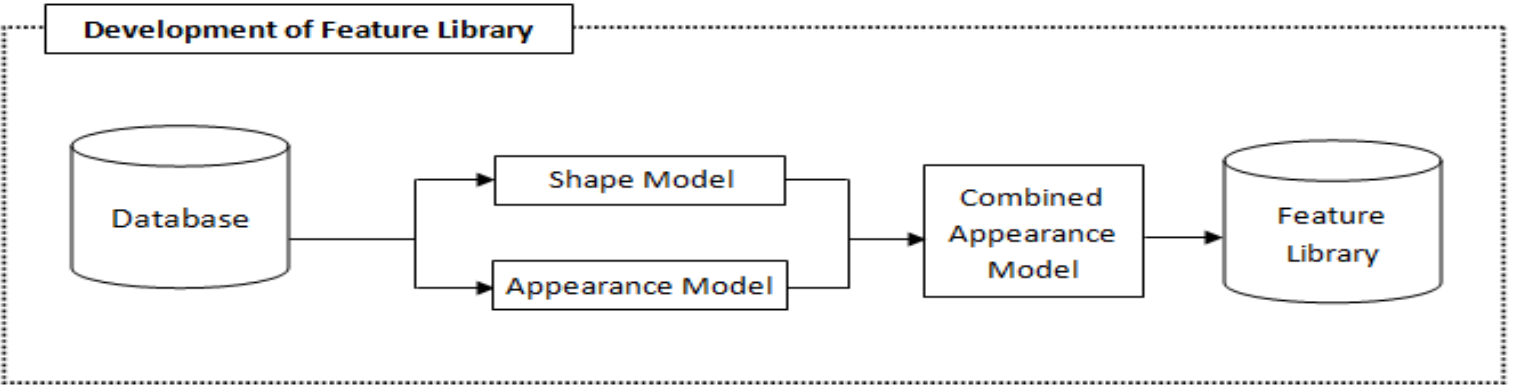

(i)

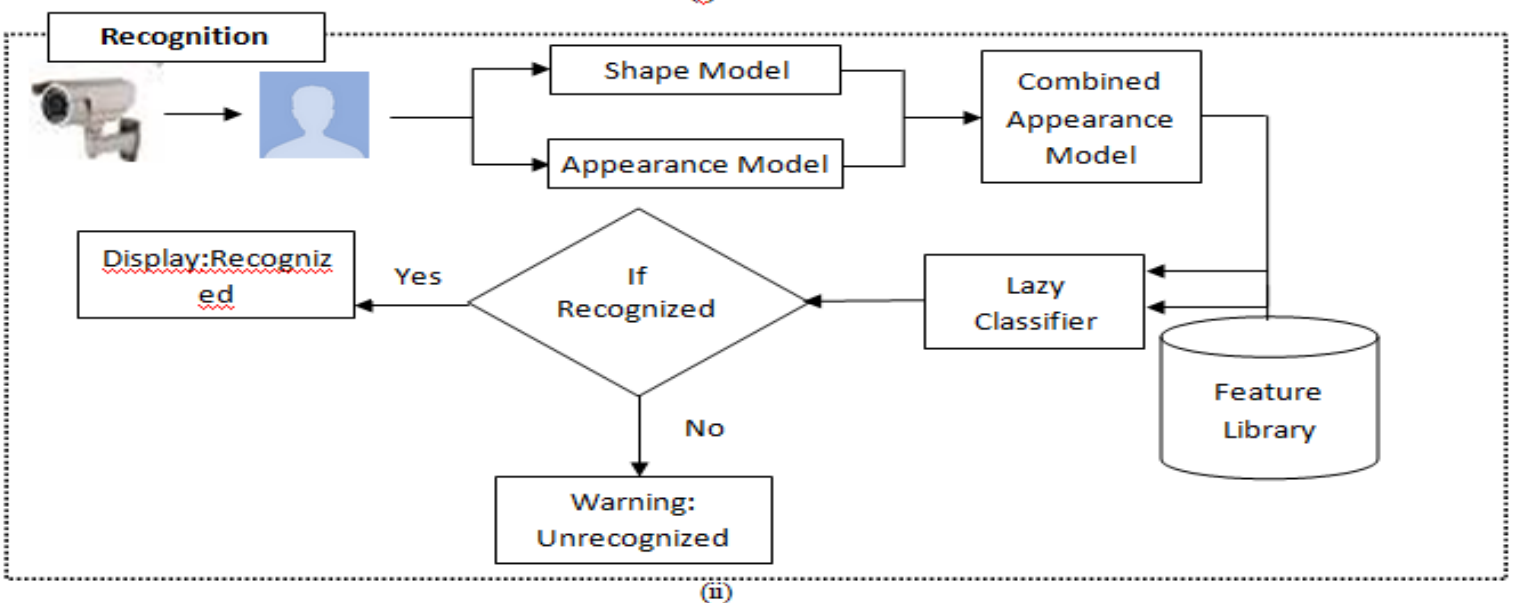

Figure 1: High Level Block Diagram of Proposed Face Recognition (i) Development of Feature Library and (ii) Recognition Stage

\section{A. Development of Feature Library}

Let us consider a facial database $V=\left\{V_{i} \mid i=1, \cdots, N\right\}$, where $N$ is the number of videos in the database. In the database, $V_{i}$ is $i^{\text {th }}$ video, which is of length $T$ seconds and holds $N_{p}$ number of poses. For every pose, a sample frame $f_{i p}: p=0,1, \ldots, N_{p}-1$ is selected and then the combined appearance model is extracted. In order to extract a combined appearance model for every $i^{\text {th }}$ video, individual shape and appearance models are extracted and subsequent combining processing is performed. In both the shape and appearance model extraction, manual intervention is required as like in the AAM, in which the active portions of faces are manually marked and then they are subjected to shape and appearance model extraction. The manually marked active portions of some sample faces are given in Figure 2.

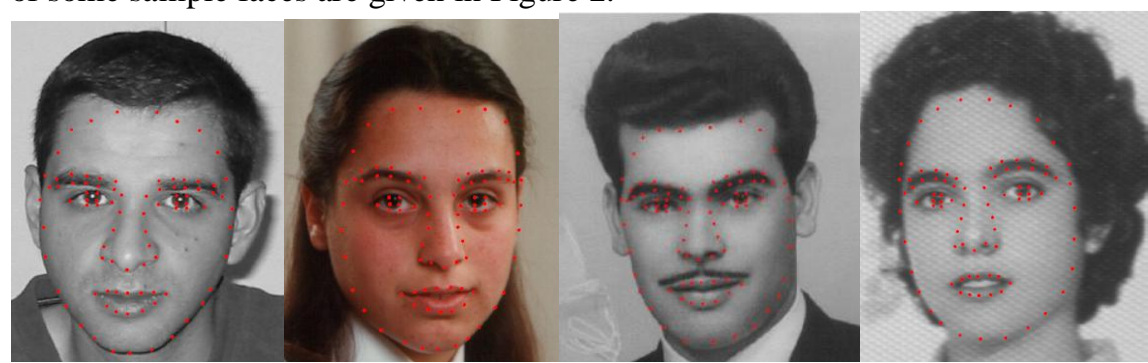

Figure 2: Active marks on sample images

\section{B. Shape Model Extraction}

Generally, shape model is a statistical model, which depicts the shape features of a deformable object. Conventional shape model [26] performs iterative procedures to determine precise statistical relationship among the facial portions; however the complexity is increased because of the iterative procedure. The propose method extracts a simple shape model without any iterative procedures, which is given as follows 


$$
\begin{aligned}
& S_{i p}=\left[\begin{array}{c}
S_{i p 0} \\
S_{i p 1} \\
\vdots \\
S_{i p N_{a}-1}
\end{array}\right] \\
& \left.S_{i p q}=\left[\begin{array}{c}
S_{i p q}^{x} \\
S_{i p q}^{y}
\end{array}\right]=\left[\begin{array}{c}
E\left[\left(X_{i p q}-\mu_{i p q}^{x}\right)\left(\bar{X}_{i q}-\mu_{i p q}^{x}\right)\right. \\
E\left[\left(Y_{i p q}-\mu_{i p q}^{y}\right)\right.
\end{array}\right)\right]
\end{aligned}
$$

where, $S_{i p q}$ is the shape model of $q^{\text {th }}$ active portion of $p^{\text {th }}$ pose in $i^{\text {th }}$ video, $S_{i p q}^{x}$ and $S_{i p q}^{y}$ are the shape model of $\mathrm{x}$ and $\mathrm{y}$ co-ordinates, $X_{i p q}$ and $Y_{i p q}$ are a vector of $\mathrm{x}$ co-ordinates of $q^{\text {th }}$ active portion of $p^{\text {th }}$ pose in $i^{\text {th }}$ video, respectively, $\mu_{i q}^{x}, \mu_{i q}^{y}, \bar{X}_{i q}$ and $\bar{Y}_{i q}$ are the local mean and global mean shape model of $q^{\text {th }}$ active portion in $i^{\text {th }}$ video, respectively. The local mean and global mean shape models can be determined as follows

$$
\begin{aligned}
& \bar{X}_{i q}=\frac{1}{N_{p}} \sum_{p=0}^{N_{p}-1} X_{i p q} \\
& \mu_{i q}=0.5\left(X_{i p q}+X_{i q}\right)
\end{aligned}
$$

\section{Appearance Model Extraction}

Active Appearance Model is a statistical model that defines shape and appearance of an object. In our work, we exploit the benefits of appearance model simply by considering the gray levels of the interconnected landmark points. An interconnection of land mark points that depict appearance of a face is shown below.
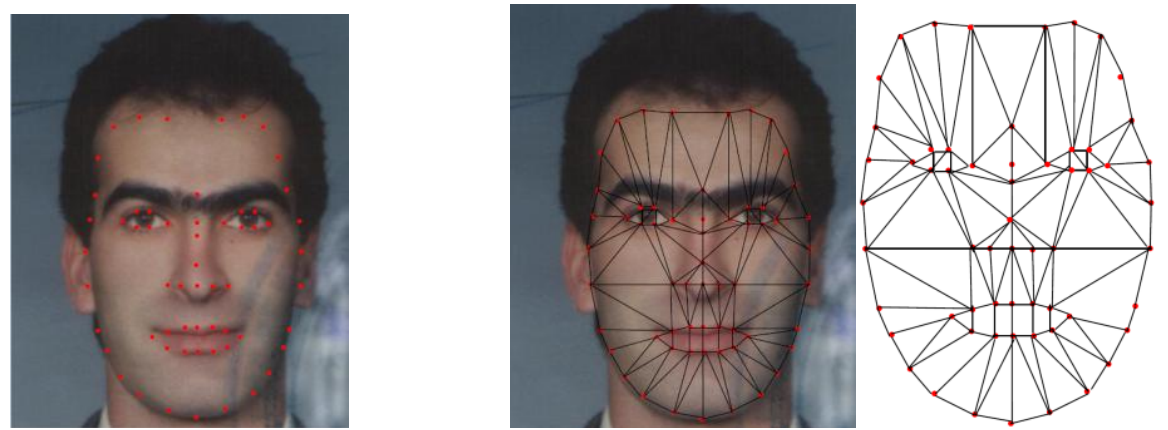

Figure 3: Extraction of Appearance Model (i) Face with Landmark points, (ii) Mesh structure Landmark points $A_{i p}=\left[\begin{array}{c}A_{i p 0} \\ A_{i p 1} \\ \vdots \\ A_{i p N_{a}-1}\end{array}\right]$ and (iii) Mesh Model of Face Appearance

From figure 3 , it can be seen that an appearance structure of the face is firstly landmarked, secondly a mesh structure is formed and eventually, the mesh model is extracted. The position of the such mesh nodes are considered as shape model in the previous Section and the gray portions are considered as the appearance model along with the interconnect information. The extracted appearance model can be represented as in equation (5) in which $A_{i p} \in A_{i p q}\left(S_{i p}\right)$

\section{Recognition Stage}

The Recognition stage performs most of the similar processes as done in the feature library and then performs a lazy classification to recognize the acquired face belongs to an authorized person or not. The recognition systems may either work in online or in offline, anyhow the face is acquired by video acquisition system and the face localization is performed. Once the localized face is grabbed, then the further process of extracting the 
features takes place. As stated earlier, the further extraction of facial shape and appearance model takes as performed in Section B \& C and test feature set is determined as follows.

\section{E. Lazy Classification}

This work exploits lazy classification as because the lazy classification requires no training process, which can highly reduce the system complexity. Despite lazy classification is not assured for recognition accuracy, the features are extracted in such a way that improved recognition accuracy can be accomplished from the introduced lazy classifier. The lazy classification process is performed with the aid of Squared Euclidean Distance (SED). The classification model is given as follows

$$
\gamma=\underset{i}{\arg \min } \frac{N D_{i}^{S} D_{i}^{A}}{\sum_{i=0}^{N-1}\left(D_{i}^{S} D_{i}^{A}\right)}: \gamma<\gamma_{T}
$$

where,

$$
\begin{gathered}
D_{i}^{S}=\sqrt{\sum_{p=0}^{N_{p}-1} \sum_{q=0}^{N_{a}-1}\left(S_{i p q}-S_{q}^{\text {test }}\right)^{2}} \\
D_{i}^{A}=\sqrt{\sum_{p=0}^{N_{p}-1} \sum_{q=0}^{N_{a}-1}\left(A_{i p q}-A_{q}^{\text {tset }}\right)^{2}}
\end{gathered}
$$

In equation (6), $\gamma$ is the classification distance, $\gamma_{T}$ is the distance threshold, $D_{i}^{S}$ and $D_{i}^{A}$ are the shape and correlation parameters, respectively.

As per the model, which is given in equation (6), the classification is performed and the face shall be a recognized face, whereas if $\gamma=\phi$ decision would be unrecognized. Eventually a recognized face is identified for its identification number and the approved credentials are displayed through output device.

\section{Experimental Results}

The proposed face recognition technique is implemented in the working platform of MATLAB (version 7.11) and tested in a machine, which has Intel Core i5 Processor, 4GB RAM and a clock speed of 3.20 GHz. The performance of the proposed technique is analyzed using UPC Face Database. For simplicity and visualize the performance in depth, we divide the database into four subsets and we organize in such a way that each subset has different pose and illumination views of ten persons. In every database, ten-fold cross-validation is performed and the performance is observed in terms of statistical measures and computational time.

\section{A. Performance Analysis}

The cross-validation results of the proposed and conventional face recognition technique [27] on the benchmark datasets are given as confusion matrices and statistical measures in Table I and II, respectively.

TABLE I: Confusion Matrices of proposed and conventional methods for (i) Dataset 1, (ii) Dataset 2, (iii) Dataset 3 and (iv) Dataset 4

(i)

\section{(a)Proposed Method (b) Conventional Method}

\begin{tabular}{|l|l|}
\hline 4 & 0 \\
\hline 1 & 5 \\
\hline
\end{tabular}

\begin{tabular}{|l|l|}
\hline 2 & 0 \\
\hline 3 & 5 \\
\hline
\end{tabular}


(ii)

(a)Proposed Method (b) Conventional Method

\begin{tabular}{|l|l|}
\hline 4 & 1 \\
\hline 1 & 4 \\
\hline
\end{tabular}

\begin{tabular}{|l|l|}
\hline 1 & 1 \\
\hline 4 & 4 \\
\hline
\end{tabular}

(iii)

(a)Proposed Method (b) Conventional Method

\begin{tabular}{|l|l|}
\hline 3 & 1 \\
\hline 2 & 4 \\
\hline
\end{tabular}

\begin{tabular}{|l|l|}
\hline 1 & 3 \\
\hline 4 & 2 \\
\hline
\end{tabular}

(a)Proposed Method (b) Conventional Method

(iv)

\begin{tabular}{|l|l|}
\hline 3 & 1 \\
\hline 2 & 4 \\
\hline
\end{tabular}

\begin{tabular}{|l|l|}
\hline 1 & 0 \\
\hline 4 & 5 \\
\hline
\end{tabular}

TABLE II: Cross-validation results between proposed and conventional face recognition systems on (i) Dataset I, (ii) Dataset II, (iii) Dataset III and (iv) Dataset IV

\begin{tabular}{|l|l|l|}
\hline Performance Metrics & Proposed Method & Conventional Method \\
\hline Accuracy (in \%) & 90 & 70 \\
\hline Sensitivity (in \%) & 80 & 40 \\
\hline Specificity (in \%) & 100 & 100 \\
\hline False Positive Rate (FPR) (in \%) & 0 & 0 \\
\hline $\begin{array}{l}\text { Positive Predictive Value (PPV) (in } \\
\% \text { ) }\end{array}$ & 100 & 100 \\
\hline $\begin{array}{l}\text { Negative Predictive Value (NPV) } \\
\text { (in \%) }\end{array}$ & 83.3 & 62.5 \\
\hline False Discovery Rate (FDR) (in \%) & 0 & 0 \\
\hline $\begin{array}{l}\text { Mathew Correlation Coefficient } \\
\text { (MCC) }\end{array}$ & 0.82 & 0.5 \\
\hline
\end{tabular}

(i) 


\begin{tabular}{|l|l|l|}
\hline Performance Metrics & Proposed Method & Conventional Method \\
\hline Accuracy (in \%) & 80 & 50 \\
\hline Sensitivity (in \%) & 80 & 20 \\
\hline Specificity (in \%) & 80 & 80 \\
\hline False Positive Rate (FPR) (in \%) & 20 & 20 \\
\hline $\begin{array}{l}\text { Positive Predictive Value (PPV) (in } \\
\% \text { ) }\end{array}$ & 80 & 50 \\
\hline $\begin{array}{l}\text { Negative Predictive Value (NPV) } \\
\text { (in \%) }\end{array}$ & 80 & 50 \\
\hline False Discovery Rate (FDR) (in \%) & 20 & 50 \\
\hline $\begin{array}{l}\text { Mathew Correlation Coefficient } \\
\text { (MCC) }\end{array}$ & 0.48 & 0 \\
\hline
\end{tabular}

(ii)

\begin{tabular}{|l|l|l|}
\hline Performance Metrics & Proposed Method & Conventional Method \\
\hline Accuracy (in \%) & 70 & 30 \\
\hline Sensitivity (in \%) & 60 & 20 \\
\hline Specificity (in \%) & 80 & 40 \\
\hline False Positive Rate (FPR) (in \%) & 20 & 60 \\
\hline $\begin{array}{l}\text { Positive Predictive Value (PPV) (in } \\
\% \text { ) }\end{array}$ & 75 & 25 \\
\hline $\begin{array}{l}\text { Negative Predictive Value (NPV) (in } \\
\%)\end{array}$ & 66.66 & 33.33 \\
\hline False Discovery Rate (FDR) (in \%) & 25 & 75 \\
\hline $\begin{array}{l}\text { Mathew Correlation Coefficient } \\
\text { (MCC) }\end{array}$ & 0.3265 & -0.163 \\
\hline
\end{tabular}

(iii)

\begin{tabular}{|l|l|l|}
\hline Performance Metrics & Proposed Method & Conventional Method \\
\hline Accuracy (in \%) & 70 & 60 \\
\hline Sensitivity (in \%) & 60 & 20 \\
\hline Specificity (in \%) & 80 & 100 \\
\hline False Positive Rate (FPR) (in \%) & 20 & 0 \\
\hline $\begin{array}{l}\text { Positive Predictive Value (PPV) (in } \\
\% \text { ) }\end{array}$ & 75 & 100 \\
\hline $\begin{array}{l}\text { Negative Predictive Value (NPV) } \\
\text { (in \%) }\end{array}$ & 66.66 & 55.55 \\
\hline False Discovery Rate (FDR) (in \%) & 25 & 0 \\
\hline $\begin{array}{l}\text { Mathew Correlation Coefficient } \\
\text { (MCC) }\end{array}$ & 0.326599 & 0.3333 \\
\hline
\end{tabular}

(iv) 


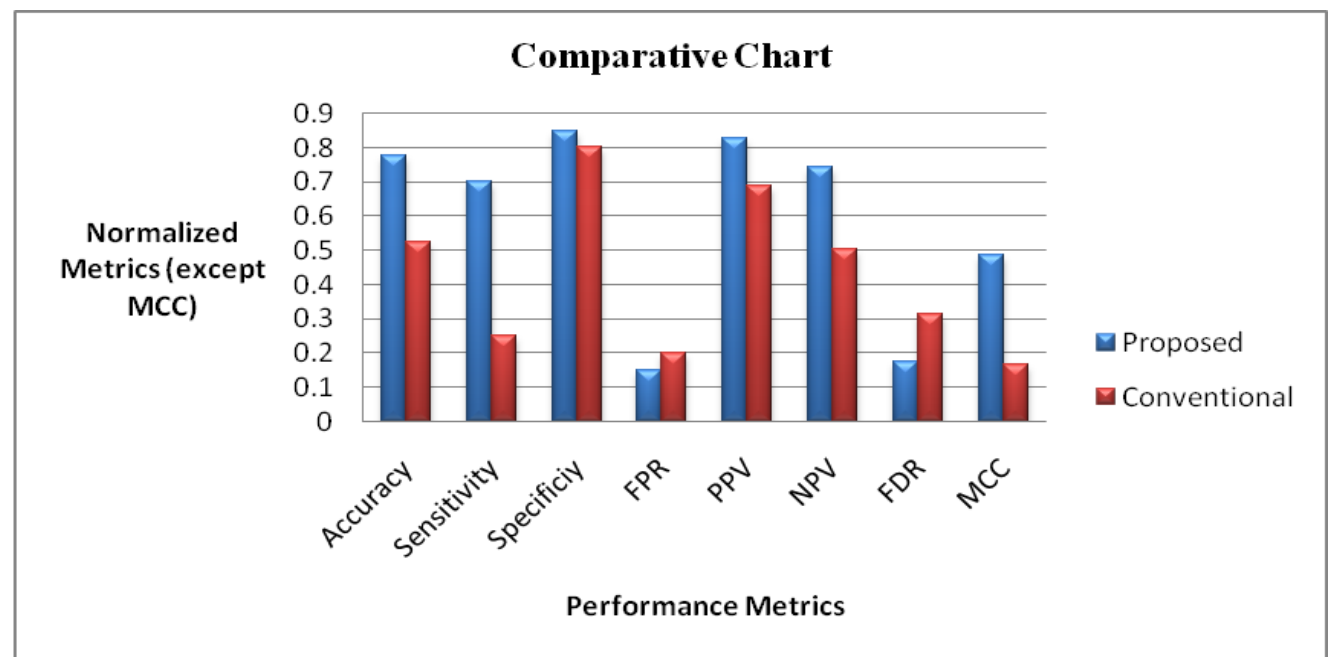

Figure 4: Averaged Comparative Chart between the proposed and conventional face recognition technique

In each confusion matrix (Table 1), the top left and right represents True positive (TP) and False positive (FP), respectively and bottom left and right represents False Negative (FN) and True Negative (TN) respectively. Here, the TP, FP, TN and FN values are determined as follows

$\mathrm{TP}$ - when an authorized person is identified correctly

FP - when an unauthorized person is identified as authorized

$\mathrm{FN}$ - when an authorized person is identified as unauthorized

TN - when an unauthorized person is identified correctly

Based on the confusion matrices, the statistical performance measures such as accuracy, sensitivity, specificity, FPR, PPV, NPV, FDR and MCC are calculated and tabulated in Table II. In most cases, the performance of the proposed technique is better than the conventional face recognition technique. Even in some instants, the conventional face recognition technique is outperforming rather than the proposed technique, for instance, for dataset 4 , PPV of proposed method is only $75 \%$ of the conventional method, but when averaged data is considered, the proposed technique outperforms. This can be visualized from the comparative chart, which is illustrated in figure 4.

\section{B. Complexity Analysis}

The proposed face recognition technique is computationally less complex rather than the conventional face recognition technique despite pose and illumination factors are considered in the process. The determined computational time for testing is tabulated in Table III and the averaged comparative chart is given in Figure 5.

Table III: Time taken by the recognition stage of proposed and conventional method under various crossvalidation experiments

\begin{tabular}{|l|l|l|}
\hline $\begin{array}{l}\text { Experiment } \\
\text { No. }\end{array}$ & Proposed Method (in seconds) & Conventional Method (in seconds) \\
\hline 1 & 0.046403 & 9.132254 \\
\hline 2 & 0.045505 & 7.777212 \\
\hline 3 & 0.046203 & 8.586688 \\
\hline 4 & 0.048359 & 4.722249 \\
\hline 5 & 0.047265 & 8.109439 \\
\hline 6 & 0.046123 & 8.615386 \\
\hline 7 & 0.043215 & 8.498807 \\
\hline 8 & 0.048245 & 8.135393 \\
\hline 9 & 0.049569 & 8.639124 \\
\hline 10 & 0.049575 & 9.459281 \\
\hline
\end{tabular}

(i) 
A Fast Recognition Method for Pose and Illumination Variant Faces on Video Sequences

\begin{tabular}{|l|l|l|}
\hline $\begin{array}{l}\text { Experiment } \\
\text { No. }\end{array}$ & Proposed Method (in seconds) & Conventional Method (in seconds) \\
\hline 1 & 0.003829 & 8.87961 \\
\hline 2 & 0.003719 & 8.208001 \\
\hline 3 & 0.003982 & 9.501837 \\
\hline 4 & 0.003579 & 7.729446 \\
\hline 5 & 0.003721 & 8.624749 \\
\hline 6 & 0.003845 & 7.71216 \\
\hline 7 & 0.003956 & 7.793763 \\
\hline 8 & 0.003485 & 9.518724 \\
\hline 9 & 0.003614 & 9.740584 \\
\hline 10 & 0.003525 & 8.790306 \\
\hline
\end{tabular}

(ii)

\begin{tabular}{|l|l|l|}
\hline $\begin{array}{l}\text { Experiment } \\
\text { No. }\end{array}$ & Proposed Method (in seconds) & Conventional Method (in seconds) \\
\hline 1 & 0.003693 & 7.150357 \\
\hline 2 & 0.003591 & 7.618764 \\
\hline 3 & 0.003489 & 7.200222 \\
\hline 4 & 0.003562 & 7.734955 \\
\hline 5 & 0.003548 & 7.60312 \\
\hline 6 & 0.003674 & 7.234343 \\
\hline 7 & 0.003697 & 7.497155 \\
\hline 8 & 0.003789 & 7.594407 \\
\hline 9 & 0.003815 & 7.1227 \\
\hline 10 & 0.003835 & 7.281026 \\
\hline
\end{tabular}

(iii)

\begin{tabular}{|l|l|l|}
\hline $\begin{array}{l}\text { Experiment } \\
\text { No. }\end{array}$ & Proposed Method (in seconds) & Conventional Method (in seconds) \\
\hline 1 & 0.003914 & 33.945641 \\
\hline 2 & 0.003819 & 24.823843 \\
\hline 3 & 0.003818 & 25.969141 \\
\hline 4 & 0.003958 & 36.278052 \\
\hline 5 & 0.003882 & 33.465799 \\
\hline 6 & 0.004015 & 44.872417 \\
\hline 7 & 0.003614 & 33.945641 \\
\hline 8 & 0.003689 & 24.823843 \\
\hline 9 & 0.003715 & 38.945641 \\
\hline 10 & 0.003769 & 28.823843 \\
\hline
\end{tabular}

(iv)

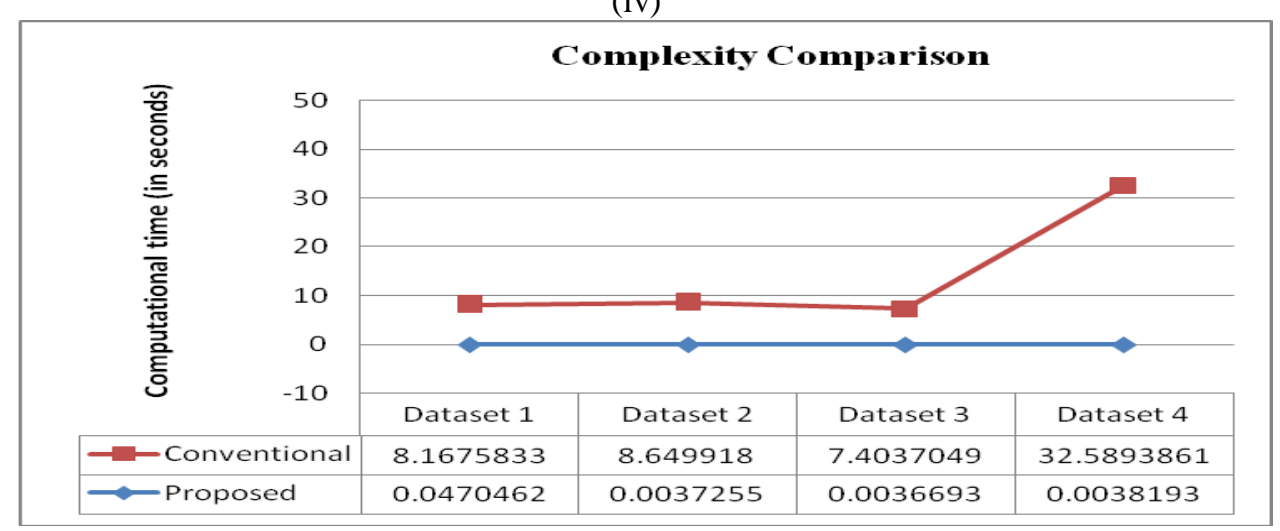

Figure 5: Averaged complexity comparison on proposed and conventional face recognition method 
From Table III and Figure 5, it can be seen that the proposed face recognition technique is very less complex than the conventional face recognition technique. The comparison shows that there is tens of deviation between them. In order further substantiate, averaged complexity is determined in which tens of deviation can be seen for datasets 1, 2 and 3 and three tens of deviation for dataset 4. Moreover, proposed technique consumes consistent time duration for evaluating an unknown image, which is an added advantage over the conventional recognition method.

\section{Conclusion}

This paper introduced a simple face recognition method to perform precise recognition in least computational cost. The work intended to solve the drawback of high computational cost of our previous recognition method. The experimental results showed that the proposed method outperforms the previous work in terms of both recognition accuracy and computational cost. This is mainly because of the fact that the usage of appearance model parameters in a simple way without exploiting any nonlinear programming. Moreover, the lazy classification deployed in the method further reduced the computational complexity. Even though, the work was mainly focused on reducing the computational complexity on recognition stage, there could not be any compromise on the precision of recognition. However, as the analysis was made on a limited datasets and not in a common bench, the future scope of the work relies on careful and extensive analysis over the technique under various test setups.

\section{REFERENCES}

[1] Wu-Jun Li, Chong-Jun Wang, Dian-Xiang Xu and Shi-Fu Chen, "Illumination Invariant Face Recognition Based on Neural Network Ensemble", In Proceedings of 16th IEEE International Conference on Tools with Artificial Intelligence, pp. 486-490, November 2004.

[2] Majumdar and Ward, "Pseudo-Fisher face Method for Single Image Per Person Face Recognition", In Proceedings of IEEE International Conference on Acoustics, Speech and Signal Processing, Las Vegas, NV, pp. 989-992, 2008.

[3] GenciCapi, "A Robotic System for Intelligent Real Time Face Recognition", ICGST International Journal on Automation, Robotics and Autonomous Systems, Vol. 9, No. 1, pp. 25-29, July 2009.

[4] Rehab F. Abdel-Kader, Rabab M. Ramadan and Rawya Y. Rizk, "Rotation Invariant Face Recognition Based on Hybrid LPT/DCT Features", International Journal of Electrical and Computer Engineering, Vol. 3, No. 7, pp. 488-493, 2008.

[5] Shaohua Kevin Zhou and Rama Chellappa, "Image-based face recognition under illumination and pose variations", Journal of the Optical Society of America A, Vol. 22, No. 2, pp. 217-229, 2005.

[6] Brunelli and Poggio, "Face recognition: Features versus templates", IEEE Transactions on Pattern Analysis and Machine Intelligence, Vol. 15, No. 10, pp.1042-1052, 1993.

[7] LaurenzWiskott, Jean-Marc Fellous, Norbert Kruger, and Christoph von der Malsburg, "Face Recognition by Elastic Bunch Graph Matching", IEEE Transactions on Pattern Analysis and Machine Intelligence, Vol.19, pp. 775-779, 1997.

[8] Edwards, Cootes and Taylor, "Face recognition using active appearance models", In Proceedings of the 5th European Conference on Computer Vision, Vol. 2, Freeburg, Germany,pp. 581-595, 1998.

[9] Zhao, Chellappa, Phillips and Rosenfeld, " Face Recognition: A Literature survey", Journal of ACM Computing Surveys, Vol. 35, No. 4, pp. 1-72, December 2003.

[10] SushmaJaiswal, Sarita Singh Bhadauria, Rakesh Singh Jadon and Tarun Kumar Divakar, "Brief description of image based 3D face recognition methods", 3D Research, Vol. 1, No. 4, pp. 1-15, 2010.

[11] Erdogan, Ercil, Ekenel, Bilgin, Eden, Kirisci and Abut, "Multi-modal Person Recognition for Vehicular Applications", In Multiple Classifier Systems, Vol. 3541, pp. 366-375, 2005.

[12] SeokCheolKee, Kyoung Mu Lee And Sang Uk Lee, " Illumination Invariant Face Recognition Using Photometric Stereo", IEICE Transactions on Information and Systems, Vol. E83-D, No. 7, pp. 1466-1474, July 2000.

[13] Yasufumi Suzuki and Tadashi Shibata, "Illumination-Invariant Face Identification Using Edge-Based Feature Vectors In Pseudo-2d Hidden Markov Models", In Proceedings of the 14th European Signal Processing Conference, Florence, Italy, 2006

[14] Hui-Fuang Ng, "Pose-Invariant Face Recognition Security System", Asian Journal of Health and Information Sciences, Vol. 1, No. 1, pp. 101-111, 2006.

[15] Shermina, "Impact of Locally Linear Regression and Fisher Linear Discriminant Analysis in Pose Invariant Face Recognition", International Journal of Computer Science and Network Security, VOL.10 No.10, pp. 106-110, October 2010.

[16] Hui-Fuang and Hong-Wen Chen, "Pose and expression invariant face recognition from a single training sample using similarity vector", Journal of Cybernetics and Systems, Vol. 1, No. 1, pp. 21-26, 2008.

[17] Wu-Jun Li, Bin Luo, Chong-Jun Wang, Xiang-Ping Zhong and Zhao-Qian Chen, "A Multiple Eigenspaces Constructing Method and Its Application to Face Recognition", In Proceedings of First International Conference on Advances in Natural Computation, Vol. 3611, pp.55-64, 2005.

[18] S.Venkatesan, S.SrinivasaRaoMadane,"Face Recognition System with Genetic Algorithm and ANT Colony Optimization", International Journal of Innovation, Management and Technology, Vol. 1, No. 5, December 2010.

[19] YileiXu, Amit Roy-Chowdhury, and KeyurPatel,"Integrating Illumination, Motion, and Shape Models for Robust Face Recognition in Video", EURASIP Journal on Advances in Signal Processing, Vol. 2008,pp 13,2007.

[20] Connolly, J.F., Granger, É. and Sabourin, R., “An Adaptive Classification System for Video-Based Face Recognition”, Information Sciences, In Press, doi:10.1016/j.ins.2010.02.026, march 2010.

[21] Unsang Park and Anil K. Jain," Face Recognition in Video: Adaptive Fusion of Multiple Matchers", IEEE computer society workshop on bio metrics, Minnerpolis, USA, June 2007.

[22] Xiujuan Chai, Shiguang Shan," Locally Linear Regression for Pose-InvariantFace Recognition", IEEE transaction on image processing, Vol. 16, No. 7, pp. 1716-1725,July 2007. 
[23] OgnjenArandjelovic, Roberto Cipolla," A methodology for rapid illumination-invariant face recognition using image processing filters", ELSEVIER, PP. 159-171, 2009.

[24] Zafar g. Sheikh, thakarev.m. and sherekars.s, "Towards Retrieval of Human Face from Video Database: A Novel Framework", Journal of Information Systems and Communication, Volume 3, Issue 1, pp.154-157, 2012

[25] Z G Sheikh, V M Thakare and S SSherekar, "Methodology for Human Face retrieval from video sequences based on holistic approach", IJCA Proceedings on National Conference on Innovative Paradigms in Engineering and Technology (NCIPET 2012) ncipet(11):7-11, March 2012

[26] T. F. Cootes, C. J. Taylor, D. H. Cooper and J. Graham, “Active Shape Models: Their Training and Application” Computer Vision and Image Understanding, Vol. 61, No. 1, p.p. 38-59, 1995

[27] T. Shreekumar and Nagarathna Hedge, "Pose and Illumination Invariant Video Face Recognition Technique: An Integrative Approach", in communication with International Journals 